\title{
Slow Wave Activity and Modulations in Mouse Jejunum Myenteric Plexus In Situ
}

\author{
Ying Cai, He Tang, Fan Jiang, and Zhaojun Dong* \\ Institute of Toxicology, College of Preventive Medicine, Third Military Medical University, Chongqing, China
}

\section{Background/Aims}

Myenteric plexus interstitial cells of Cajal (ICC-MY) are involved in the generation of gut pacemaker activity and neuronal communication. We performed patch clamp on ICC-MY in situ to observe the changes of pacemaker activity in response to neural modulations.

\section{Methods}

A fresh longitudinal muscle with myenteric plexus (LMMP) from mouse jejunum was prepared. ICC-MY and ganglion neurons embedded in the layer of longitudinal muscles were targeted by patch clamping in whole-cell configuration in a model of current or voltage clamp. Neurogenic modulators were applied to evaluate their effects on ICC pacemaker activity.

\section{Results}

In situ ICC-MY showed spontaneous and rhythmical voltage oscillations with a frequency of $27.2 \pm 3.9$ cycles/min, amplitude of $32.6 \pm 6.3 \mathrm{mV}$, and resting membrane potential of $-62.2 \pm 2.8 \mathrm{mV}$. In situ neurons showed electrically evocable action potential in single or multiple spikes. Pacemaker activity was modulated by neuronal activators through receiving a neuronal input. Application of tetrodotoxin depolarized pacemaker potentials in a dose dependent manner, and decreased the amplitude at tetrodotoxin $0.3 \mu \mathrm{M}$ for about $40 \pm 10 \%$; capsaicin $(1 \mu \mathrm{M})$ ameliorated ICC-MY K+ current for about $49 \pm 14.8 \%$; and, nitric oxide hyperpolarized pacemaker potential and decreased the amplitude and frequency.

\section{Conclusions}

The in situ preparation patch clamp study further demonstrates that the pacemaker activity is an intrinsic property of ICC. The neurogenic activators change and shape pacemaker potential and activity in situ. LMMP preparation in situ patch clamp provides an ideal platform to study the functional innervation of the ICC and the enteric neural system, thereby, for evaluating the neural regulation of pacemaker activity, especially in disorder models.

(J Neurogastroenterol Motil 2017;23:117-123)

\section{Key Words}

Enteric nervous system; In situ patch clamp; Interstitial cells of Cajal; Pacemaker activity

Received: January 21, 2016 Revised: May 23, 2016 Accepted: June 6, 2016

(c) This is an Open Access article distributed under the terms of the Creative Commons Attribution Non-Commercial License (http://creativecommons. org/licenses/by-nc/4.0) which permits unrestricted non-commercial use, distribution, and reproduction in any medium, provided the original work is properly cited.

*Correspondence: Zhaojun Dong, PhD

College of Preventive Medicine, Third Military Medical University, Chongqing 400038, China

Tel: +86-23-68772390, Fax: +86-23-68772390, E-mail: dongzj@163.com 


\section{Introduction}

The gut musculature is the wall integrated with the enteric nervous system and interstitial cells of the Cajal (ICC). In the small intestine, ICC associated with the myenteric plexus lie between the circular and longitudinal smooth muscle layers. These ICC, instead of neurons or smooth muscles, generate rhythmical pacemaker activity that drives slow waves along smooth muscles for peristalsis. ${ }^{1}$ The structural relationship that is the myenteric plexus ICC (ICCMY) very closely surrounding the ganglion has led to questions about what is the neural role in mediation of pacemaker activity, or whether enteric neurotransmission is innervated in the pacemaker activity. In vitro patch clamp has been used in studies of ICC-MY or neurons based on their culture statuses, in which the structure of tissue organization, synaptic connections, and interactions between ICC-MY and neurons are all disrupted. Especially, ICC-MY can express different pacemaker channel or ionic conductance when the culture time and conditions are different. ${ }^{2-5}$ Similarly, cultured myenteric neurons also showed differences in ion channel complement, ${ }^{6-8}$ compared with myenteric neurons in situ. Thus, researchers have attempted to access ICC in gut tissue by a recently proposed in situ patch clamping ICC-MY or neurons from longitudinal muscle with myenteric plexus (LMMP) to explore the neural effect on ICC-MY pacemaker activity, under a natural and physiological environment. ${ }^{9-13}$ We now report our preliminary data of pacemaker activity and neuronal regulation by using the approach of in situ patch clamp in the myenteric plexus region.

\section{Materials and Methods}

\section{Myenteric Plexus Preparation}

$\mathrm{BALB} / \mathrm{C}$ mice (9-15 days old) of either sex were anaesthetized with chloroform and killed by cervical dislocation. About $10 \mathrm{~cm}$ in length of the jejunum from the pyloric ring was removed and the luminal content was flushed out with Krebs solution containing Nicardipine $(0.5 \mu \mathrm{M})$ and tetrodotoxin (TTX; $0.3 \mu \mathrm{M})$. Tissues were pinned to the base of a silicon dish, and the mucosa and submucosa were removed, then the muscle strip was cut into several pieces $(1.2 \mathrm{~cm} \times 1.2 \mathrm{~cm})$ and stored in $\mathrm{O}_{2}$ bubbled Krebs solution. The preparation was subject to further dissection before recording, ie, the circular muscle layer covering the plexus was removed to uncover 1-3 ganglions. This preparation was then placed on the stage of an inverted microscope/patch clamp setup and perfused with
Krebs solution at a constant flow rate of $2 \mathrm{~mL} / \mathrm{min}$. Toward the area to be patched, $0.1 \mathrm{mg} / \mathrm{mL}$ protease (Sigma-Aldrich, St. Louis, MO, USA) was closely flushed by for about 1 minute through a delivery tubing to create a clean surface for a giga seal.

\section{Pacemaker Cell Identification}

Pacemaker cell was identified in preparation by one or more features, including cell morphological characteristics, ${ }^{14}$ methylene blue absorption, ${ }^{15}$ and immune-reaction to fluorescein isothiocyanate (FITC) conjugated ACK2 antibody. ${ }^{5-7}$ Practically, neurons lay in a ganglion structure and their cell body are about $>15 \mu \mathrm{m}$ size, the muscle cells are buried as an integrative sheet and are lined directionally, therefore, to distinguish the glia network information from pacemaker cells is a main challenge. In our preparations, we identified in a comprehensive way including the fact that ICC were adjacent with ganglions, takes-up methylene blue, and displayed electrical pacemaker activity.

Immunohistochemical staining was performed in live tissue preparations. The rat anti-mouse CD117 antibody, clone ACK2, Alexa Fluor 488 (1:200) and polyclonal rabbit anti-neuronal nitric oxide synthase (nNOS) (1:800; Chemicon, Temecula, CA, USA) antibodies were incubated at $37^{\circ} \mathrm{C}$ with $95 \% \mathrm{O}_{2}$ and $5 \% \mathrm{CO}_{2}$ for 2 hours. After wash in shaking ( $3 \times 5$ minutes), $\mathrm{Cy} 3$ conjugated goat-anti-rabbit IgG (1:100; Jackson Immuno Research, Westgrove, PA, USA) was added for further incubation for about 30 minutes. The nitrergic neurons (red) and ICC (green) are closely positioned and both are identified for patch clamping.

\section{Patch Clamp Experiments}

Whole-cell patch clamp technique was used to record membrane potential $(\mathrm{I}=0)$ and/or currents (v-clamp) from in situ pacemaker cells. Potentials and currents were amplified with standard patch clamp amplifiers (Axopatch 100; Axon Instruments, Foster City, CA, USA) and digitized with a 12-bit A/D converter (TL-1, DMA interface, Axon Instruments). Data were filtered at $2 \mathrm{kHz}$ using an 8-pole Bessel filter. The pipette solution contained (in mM) $100 \mathrm{~K}$-aspartate, $30 \mathrm{KCl}, 5$ HEPES, 5 ATP- $\mathrm{Na}_{2}, 1 \mathrm{MgCl}_{2}$, 0.1 GTP, and 5 EGTA ( $\mathrm{pH} 7.35$ with $\mathrm{KOH}$ ). The bath was continuously superfused (in $\mathrm{mM}$ ): $\mathrm{NaCl} 118, \mathrm{KCl} 4.8, \mathrm{NaHCO}_{3}$ 25, $\mathrm{NaH}_{2} \mathrm{PO}_{4} 1.1, \mathrm{MgSO}_{4} 1, \mathrm{CaCl}_{2}$ 2, glucose 11.3 and bubbled with a mixture of $97 \% \mathrm{O}_{2}$ and $3 \% \mathrm{CO}_{2}$. The alternative solutions that were used for substitutional perfusion are detailed in the result section. The pipette tip resistance ranged between $4 \mathrm{M} \Omega$ and 7 $\mathrm{M} \Omega$. All experiments were conducted at room temperature (around $23^{\circ} \mathrm{C}$ ). Results were analyzed using pCLAMP 10 (Axon Instru- 
ments). All data are expressed as means \pm SEM. Differences in the data were evaluated by Student's $t$ test. $P$-values less than 0.05 were taken as a statistically significant difference. The $\mathrm{n}$ values reported in the text refer to the number of cells used.

\section{Results}

\section{The Longitudinal Muscle with Myenteric Plexus Preparation and Apposition of Interstitial Cells of Cajal and Neurons in Murine Jejunum In Situ}

The preparation of mouse jejunal myenteric plexus in situ, under physiological extracellular solution, was intact and rigorously contractile in a frequency of $24.5 \pm 2.5$ cycles $/ \mathrm{min}(\mathrm{n}=8)$. To achieve the ICC-MY whole cell configuration in situ, there were some practically technical difficulties that prevented the giga seal from the pipette to the cell membrane since the targeting cells were embedded in a hard-to-penetrate muscle wall, and the surface of the targeting cells was covered by connective tissues and glial cells. The accessible area of the cell was very limited, and the preparation was contracted. To overcome these limitations, a local area ready to patch was flushed with protease $(0.2 \mathrm{mg} / \mathrm{mL})$ for 1 minute before every pipette approach, nicardipine $(0.5 \mu \mathrm{M})$ was in system perfusion to suppress the contraction, ${ }^{9,10}$ and the tissue piece was re-pined or re-spread to increase or exposure the cell accessible area. Also, a thinner wall of pipette with about 1-2 $\mu \mathrm{M}$ mouth dimension was used. Despite this technique it was quite challenging and the rate of successful giga seal was much lower than that in cultured cells, it was a way to achieve high-resistance seal and whole cell configuration. ${ }^{9,10}$ Once the whole cell configuration was obtained, it was very stable and endurable for long time recording and observation.

As shown in Figure 1A, the circular muscles on the surface of ganglia were removed, the methylene blue was stained in some cells that were identified with pacemaker activity after the patch. Figure

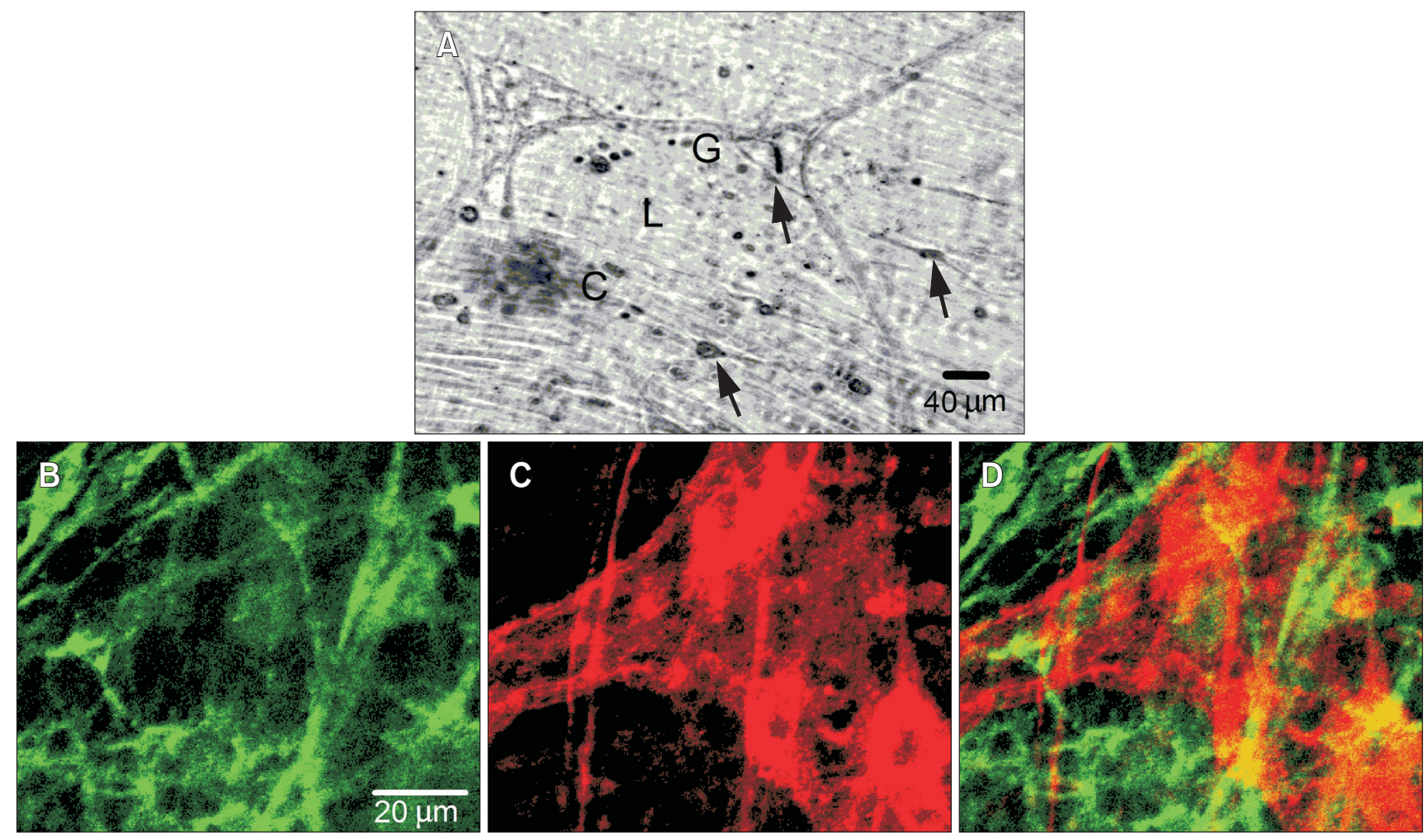

Figure 1. Identification of pacemaker cell in mouse jejunum preparation. (A) The jejunum free of mesentery, mucosa and submucosa was pinned to the silicon bottom of the Petri dish, and placed on the stage of an inverted microscope for patch clamp recording. "L" shows an area of longitudinal muscle layer on which the circular muscle " $C$ " was partially removed to expose ganglion " $G$ ". Note the exposed ganglia are on the border of circular and longitudinal muscle layers, in which the cells to be patched can have an optimal surface and access angle. The arrows show the cells that take up methylene blue and express pacemaking activity. (B-D) Antibodies of ACK2 and neuronal nitric oxide synthase live staining reveal interstitial cells of Cajal (green), ganglion neurons and fibers (red) and co-localization (orange). 
1B-D display the ACK2 and nNOS positive cells that are not colocalized or ICC-MY do not express nNOS, but ICC-MY are tightly contacted with nitrergic neurons.

A

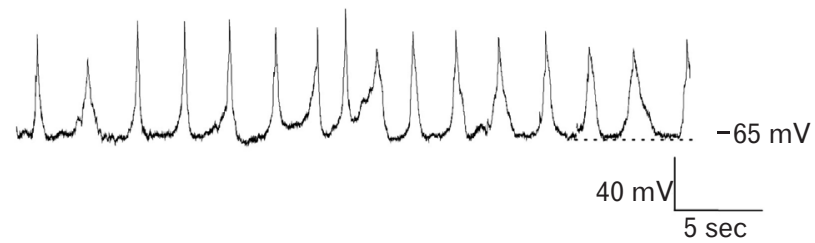

B

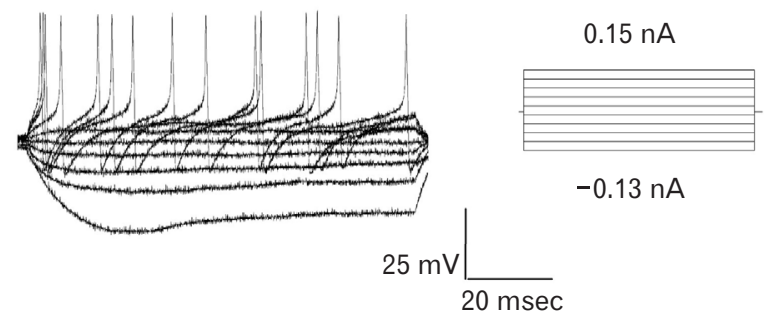

Figure 2. Spontaneous pacemaker activity and electrical evoked action potentials respectively recorded from interstitial cells of Cajal and neurons by in situ patch clamping. (A) Pacemaker potentials (slow waves) were recorded using current clamp $(\mathrm{I}=0)$. (B) Action potentials were evoked upon current injection (I-Clamp) as protocol.

\section{Spontaneous Pacemaker Potential from Interstitial Cells of Cajal and Evocable Action Potential from Neuron in Murine Jejunum In Situ}

Of the 13 ICC, with spontaneously rhythmical pacemaker activity under the current clamp ( $\mathrm{I}=0$; Fig. $2 \mathrm{~A})$, had resting membrane potentials of $-62.2 \pm 2.8 \mathrm{mV}$, capacitances of $18 \pm 3.2$ $\mathrm{pF}$, and input resistance of $260 \pm 38 \mathrm{M} \Omega$. The mean spontaneous pacemaker potentials amplitude was $32.6 \pm 6.3 \mathrm{mV}$ and the frequency was $27.2 \pm 3.9 \mathrm{cycles} / \mathrm{min}$. In a close apposition to ICC, the enteric neuron shown as an example in Figure 2B was also accessible for patch clamp and the action potentials in response to varies of current injection were obtained. Here, the traces of pacemaker activities and action potentials from in situ preparations are featured typically similar to other preparations. ${ }^{2,4,5,9,10}$

\section{The Effects of Tetrodotoxin on Spontaneous Pacemaker Activity}

Despite the well-known pacemaker activity insensitive to TTX in preparations of cell cultures and muscle strips mouse ICC-MY do not express voltage gated sodium channel $(\mathrm{NaV} 1.5),{ }^{16}$ little is known of the influence on pacemaker activity if such a neural channel is blocked in in situ physiological conditions. As shown in Figure 3A, at TTX $0.1 \mu \mathrm{M}$ the pacemaker activity was generally unaltered in amplitude, frequency, and membrane potential, except
A

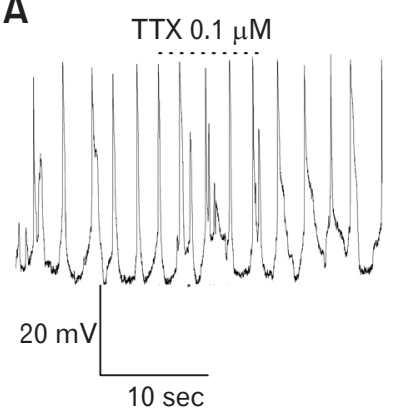

B

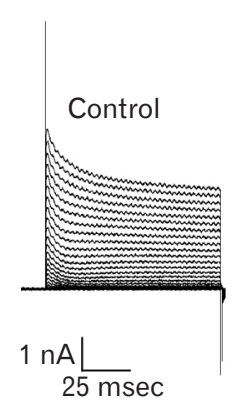

$25 \mathrm{msec}$
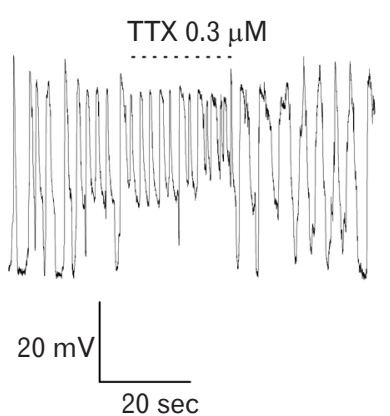

$20 \mathrm{sec}$

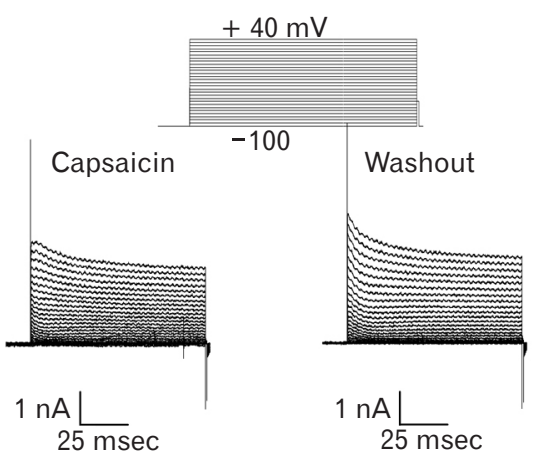

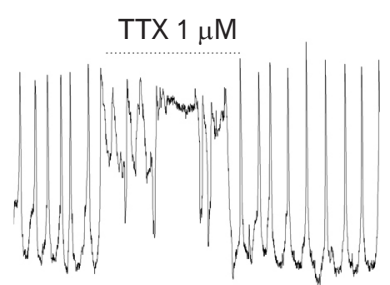

$20 \mathrm{mV} \bigsqcup_{20 \mathrm{sec}}$

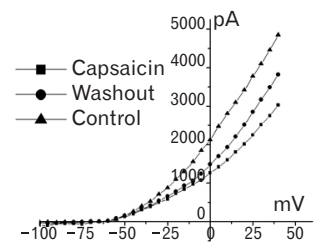

Figure 3. The modulation of pacemaker activity in response to tetrodotoxin (TTX). (A) The spontaneous pacemaker activity was expressed in response TTX at $0.1,0.3$, and $1 \mu \mathrm{M}$, respectively. The bar indicates amplitude and length. (B) The inhibition of outward $\mathrm{K}^{+}$was recorded after exposed by capsaicin $(1 \mu \mathrm{M})$ for 2 minutes; it was partially reversible after washout. Please note the extent of inhibition varies upon the exposure time. 
for the relatively increased plateau phase. However, increase of concentration to $0.3 \mu \mathrm{M}$ and $1 \mu \mathrm{M}$, the membrane potentials were significantly depolarized and the events were disrupted by over excitation. For example, at TTX $0.3 \mu \mathrm{M}$, the membrane potential of pacemaker ICC was depolarized from $-64 \pm 5.4$ to $-30 \pm 5.2 \mathrm{mV}$ ( $\mathrm{n}=4, P<0.001)$.

Since TTX depolarized slow waves, we tested if such depolarization was due to a blockage of $\mathrm{K}^{+}$current. TTX at $0.1 \mu \mathrm{M}$ and 0.3 $\mu \mathrm{M}$ did not change $\mathrm{K}^{+}$currents (data not shown), thus capsaicin was applied in for a further examination. Figure $3 \mathrm{~B}$ presents that, at voltage clamp, the typical K profile was recorded from ICC-MY. During perfusion of $1 \mu \mathrm{M}$ capsaicin, $\mathrm{K}^{+}$current was suppressed for about $47.5 \pm 11.1 \%(\mathrm{n}=5, P=0.023)$, which was reversible after withdrawn of capsaicin.

Both responses of ICC-MY to TTX and capsaicin are informative in that ICC-MY must receive inputs from neurons that

A

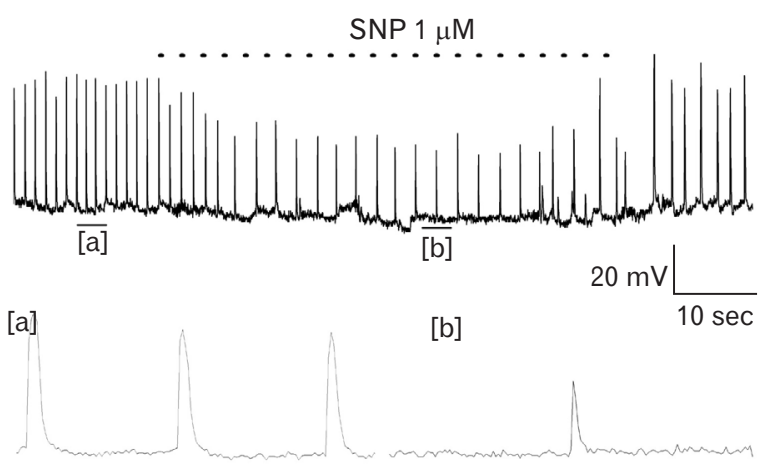

B

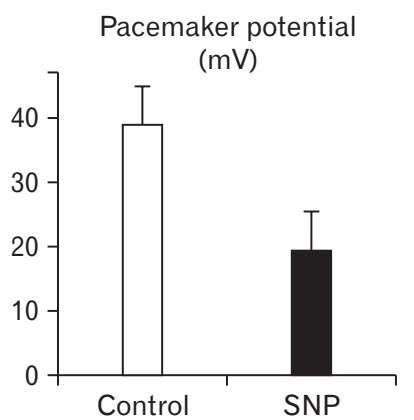

C

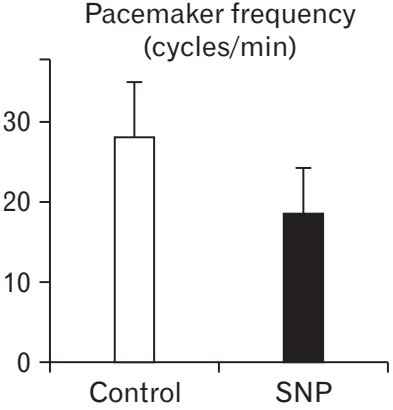

Figsure 4. Nitric oxide modulation of pacemaker activity recorded from myenteric plexus interstitial cells of Cajal immediately adjacent to neuron. (A) Spontaneous pacemaker activity was recorded in the absence and presence of sodium nitroprusside (SNP; $1 \mu \mathrm{M})$. Note the portions of the trace $[\mathrm{a}]$ and $[\mathrm{b}]$ are magnified for frequency and amplitude comparisons (B and C). A statistical data of slow waves in response to $1 \mu \mathrm{M}$ SNP from 6 different preparations respectively in amplitude and frequency. were activated corresponding to an inhibition of the $\mathrm{Na}$ channel, and release of transmitter through the transient receptor potential vanilloid type 1 (TRPV1) signal pathway since ICC-MY are unable to recognize and respond to TTX and capsaicin.

\section{Modulation of Pacemaker Potential by Nitric Oxide}

Figure 4 shows that the spontaneously rhythmical pacemaker potentials were remarkably modified after perfusing with nitric oxide (NO) donor sodium nitroprusside (SNP). SNP from 1-100 $\mu \mathrm{M}$ hyperpolarized membrane potentials and decreased the amplitude and frequency. For example, SNP $(1 \mu \mathrm{M})$ hyperpolarized membrane potentials for about $9.5 \mathrm{mV}(\mathrm{n}=6, P=0.006)$; decreased the amplitude $39 \pm 6.5 \%(\mathrm{n}=6, P=0.040)$ and the frequency $35 \pm 5.3 \%(n=6, P=0.024)$. In addition, the pacemaker activity was examined under $\mathrm{N}$-nitro-L-arginine and exhibited depolarization (data not shown), confirming a functional innervation between ICC-MY and nitrergic nerve in in situ model.

\section{Discussion}

The present study reports for the first time functional evidence of the neural regulation of the pacemaker potential with the neuronal activator and effector in in situ ICC-MY of the murine jejunum. To identify and access to ICC for whole cell configuration in the in situ patch clamp, a proper technique is critical. Due to neuron size and position that is relatively easier than ICC to locate and access, we performed the patch first onto neurons. Once having spiked, the preparation is ready for acquisition from ICC. Labeling cKit antibody may give more precise information but it increases more procedures and also affects the tissue health and pacemaker activity. ${ }^{17}$ Instead, Methylene blue staining is simple and practical since ICC can take up the dye in a short time of incubation. ${ }^{15}$

The pacemaker activity waveform obtained from in situ LMMP preparation is characteristically similar to other studies. ${ }^{2-5}$ It is established that pacemaker activity in the in situ patch clamp is feasibly obtainable. Compared to culture models, the activity recorded from in situ is more active and persistent. For instance, at room temperature rhythmic activity occurred at a frequency of 13.9 \pm 11.2 cycles $/ \mathrm{min}^{4}$, and another study showed that the spontaneous slow waves were at a frequency of $6.6 \pm 0.3$ cycles $/ \mathrm{min}$ at room temperature and the frequency increased to $13.5 \pm 0.6$ cycles $/ \mathrm{min}$ at $29^{\circ} \mathrm{C}^{2}$. The difference is possibly due to enzyme digestion difference. In a non enzyme digestion preparation, the rhythmic inward currents frequency is $20-30$ cycles/min at room temperature. ${ }^{5}$ It is clear that as the preparations are more prototype, the slow waves are 
more active.

Our interest to study in situ preparations is not only in searching for intrinsic and unique properties under a natural status, but also for activity of the ICC network and the regulation from the neighboring nerve signaling transductions. Hence, using the preparation to monitor the electrical events from neurons into ICC is an advantage.

TTX is a potent neurotoxin that blocks voltage-gated sodium channels. According to the sensitivity to this toxin, $\mathrm{Na}$ channel is classified as TTX-sensitive and TTX-resistant. Except for a report that Nav1.5 is in the presence of human ICC, ${ }^{16}$ sodium channels have not been found in ICC of murine small intestine. ICC pacemaker potential depolarized by TTX was observed in our study. Since TTX is a highly selective blocker, it is impossible that TTX directly targets onto ICC, However, in situ preparation where pacemaker activity was recorded in LMMP mimic under a tight tension condition, perhaps similar to tonic inhibition in which pacemaker activity can be synchronized by excitatory nerves to couple the longitudinal and circular muscles during motor complexes which are neutrally mediated. ICC-MY receive excitatory input from motor neurons that release acetylcholine and tachykinins acting on muscarinic and NK1 receptors, respectively. ${ }^{18-19}$ Whether or not the other effector has the same effect—like TTX — was also examined. TRPV1 is distributed in ganglion neurons, not in ICC, thus we tested if capsaicin can change the property expressed from ICC. Likewise, $\mathrm{K}^{+}$current recorded in ICC in voltage clamp can be reversibly inhibited by capsaicin. It was demonstrated that an activation of neuronal TRPV1 can affect ICC activity in the myenteric plexus. It is noted that capsaicin inhibits the spontaneous pacemaker activity in ICC, ${ }^{20}$ such a mixed culture preparation of neuron and ICC may be one of the possibilities. Nevertheless, these suggest ICC-MY and neighboring neurons are electrically coupled, and further study is required for data interpretation.

Our immunoreactivity data about the structural distribution and cellular localization of $\mathrm{nNOS}$ is consistent with data from other studies by Ward et al, ${ }^{21}$ Salmhofer et al, ${ }^{22}$ and Berezin et al, ${ }^{23}$ but different from Vannucchi et al. ${ }^{24}$ In our data, nNOS are expressed in many neurons and nerve fibers in the myenteric plexus, but not in ICC-MY. The hyperpolarization of ICC-MY in response to the NO donor SNP confirmed this relationship between NO and pacemaker activity in ICC of the mouse small intestine. It is apparent that these ICC capable producing pacemaker activities are required for innervation with the inhibitory nerves that can release NO. On the other hand, inhibition of NOS by L-NNA depolarizes the pacemaker potential. This further indicates that NOS ac- tively targets ICC to form up and regulate pacemaker activity in the mouse small intestine.

In conclusion, we have shown that the in situ preparation patch clamp can obtain ICC-MY pacemaker activity and its changes under neural regulation including NO as the regulator. Such a novel technique will contribute greatly to progress in the study of gut pacemaker cells and interactions with enteric nerves to which they are intimately connected, and further, in evaluating intestinal neuronal motility disorders.

Financial support: This work was supported by the Natural Science Foundation Project of CQ CSTC (No. cstc2015jcyjA10075).

\section{Conflicts of interest: None.}

Author contributions: Ying Cai designed and performed experiments, analyzed data, and wrote the paper; He Tang played roles in data collection and interpretation; Fan Jiang took part in the draft revising and data collection; and Zhaojun Dong contributed to the design and the revising of this manuscript, and is responsible for all aspects of the manuscript

\section{References}

1. Al-Shboul OA. The importance of interstitial cells of Cajal in the gastrointestinal tract. Saudi J Gastroenterol 2013;19:3-15.

2. Koh SD, Sanders KM, Ward SM. Spontaneous electrical rhythmicity in cultured interstitial cells of Cajal from the murine small intestine. J Physiol 1998;513(Pt 1):203-213.

3. Thomsen L, Robinson TL, Lee JC, et al. Interstitial cells of Cajal generate a rhythmic pacemaker current. Nat Med 1998;4:848-851.

4. Lee JC, Thuneberg L, Berezin I, Huizinga JD. Generation of slow waves in membrane potential is an intrinsic property of interstitial cells of Cajal. Am J Physiol 1999;277(2 Pt 1):G409-G423.

5. Huizinga JD, Zhu Y, Ye J, Molleman A. High conductance chloride channels generate pacemaker currents in interstitial cells of Cajal. Gastroenterology 2002;123:1627-1636.

6. Franklin JL, Willard AL. Voltage-dependent sodium and calcium currents of rat myenteric neurons in cell culture. J Neurophysiol 1993;69:1264-1275.

7. Starodub AM, Wood JD. Selectivity of omega-CgTx-MVIIC toxin from Conus magus on calcium currents in enteric neurons. Life Sci 1999;64:PL305-PL310.

8. Haschke G, Schafer H, Diener M. Effect of butyrate on membrane potential, ionic currents and intracellular $\mathrm{Ca}^{2+}$ concentration in cultured rat myenteric neurons. Neurogastroenterol Motil 2002;14:133-142.

9. Wang B, Kunze WA, Zhu Y, Huizinga JD. In situ recording from gut pacemaker cells. Pflugers Arch 2008;457:243-251.

10. Osorio N, Delmas P, Jones PA. Patch clamp recording from enteric neu- 
rons in situ. Nat Protoc 2011;6:15-27.

11. Winlove CI, Roberts A. The firing patterns of spinal neurons: in situ patch-clamp recordings reveal a key role for potassium currents. Eur J Neurosci 2012;36:2926-2940.

12. Nong $\mathrm{Y}$, Zhang $\mathrm{C}$, Wei L, et al. In situ investigation of allografted mouse HCN4 gene-transfected rat bone marrow mesenchymal stromal cells with the use of patch-clamp recording of ventricular slices. Cytotherapy 2013;15:905-919.

13. Zhu YF, Wang XY, Lowie BJ, et al. Enteric sensory neurons communicate with interstitial cells of Cajal to affect pacemaker activity in the small intestine. Pflugers Arch 2014;466:1467-1475.

14. Thuneberg L. One hundred years of interstitial cells of Cajal. Microsc Res Tech 1999; 47:223-238.

15. Liu LW, Thuneberg L, Daniel EE, Huizinga JD. Selective accumulation of methylene blue by interstitial cells of Cajal in canine colon. Am J Physiol 1993;264(1 Pt 1):G64-G73.

16. Beyder A, Rae JL, Bernard C, et al. Mechanosensitivity of Nav1.5, a voltage-sensitive sodium channel. J Physiol 2010;588(Pt 24):4969-4985.

17. Zhu $\mathrm{MH}$, Kim TW, Ro S, et al. $\mathrm{A} \mathrm{Ca}^{2+}$-activated $\mathrm{Cl}^{-}$conductance in interstitial cells of Cajal linked to slow wave currents and pacemaker activity. J Physiol 2009;587(Pt 20):4905-4918.

18. Smith TK, Park KJ, Hennig GW. Colonic migrating motor complexes, high amplitude propagating contractions, neural reflexes and the importance of neuronal and mucosal serotonin. J Neurogastroenterol Motil 2014;20:423-446.

19. Huizinga JD, Golden CM, Zhu Y, White EJ. Ion channels in interstitial cells of Cajal as targets for neurotransmitter action. Neurogastroenterol Motil 2004;16(suppl 1):106-111.

20. Choi S, Sun JM, Shahi PK, Zuo DC, Kim HI, Jun JY. Capsaicin inhibits the spontaneous pacemaker activity in interstitial cells of Cajal from the small intestine of mouse. J Neurogastroenterol Motil 2010;16:265-273.

21. Blair PJ, Bayguinov Y, Sanders KM, Ward SM. Relationship between enteric neurons and interstitial cells in the primate gastrointestinal tract. Neurogastroenterol Motil 2012;24:e437-e449.

22. Salmhofer H, Neuhuber WL, Ruth P, Huber A, Russwurm M, Allescher HD. Pivotal role of the interstitial cells of Cajal in the nitric oxide signaling pathway of rat small intestine. Morphological evidence. Cell Tissue Res 2001;305:331-340.

23. Berezin I, Snyder SH, Bredt DS, Daniel EE. Ultrastructural localization of nitric oxide synthase in canine small intestine and colon. Am J Physiol 1994;266(4 Pt 1):C981-C989.

24. Vannucchi MG, Corsani L, Bani D, Faussone-Pellegrini MS. Myenteric neurons and interstitial cells of Cajal of mouse colon express several nitric oxide synthase isoforms. Neurosci Lett 2002;326:191-195. 